\title{
Prolapsing Urethral Rhinosporidiosis
}

\section{Dilip Kumar Pal ${ }^{\star}$}

Department of Urology, Institute of Post Graduate Medical Education \& Research, Kolkata, India-700020

\begin{abstract}
Rhinosporidiosis, a rare chronic granulomatous condition caused by a fungus, Rhinosporidium seeberi. Usually it affects the nasal mucosa, urethra is rare site involved in this disease. Here we are reporting a rare case where rhinosporidiosis presented as a protruding mass from urethra during voiding.
\end{abstract}

Keywords: Rhinosporidiosis; Urethral; Genito-urinary; Prolapsing urethral mass

\section{Introduction}

Rhinosporidiosis, first described by Gulleimaro Seeberi from Buenos Aires in 1900 [1], is a rare chronic granulomatous condition usually affecting the anterior nares, caused by a fungus Rhinosporidiosisseeberi [1,2]. Ashworth in 1923 described the lifecycle of this fungus and established the name [1]. Though it is globally distributed but $90 \%$ cases are from Asia mainly from South India, Srilanka, Pakistan and less than $5 \%$ case are from Africa \& western countries [3]. The nasopharynx and nose (nasal mucosa) commonly (70\%) affected in this disease but conjunctiva, lachrymal sac, lip, palate, skin, larynx, trachea, bronchi, vagina and vulva are the other sites which may be affected [2-4]. Urethra may be involved but it is very rare, only few cases reported in literature till date and they are mostly from India [3-5]. Here we report a case of urethral rhinosporidiosis protruding from external urethral meatus during voiding.

\section{Case Report}

A 35 year old man from rural tribal background, farmer by occupation presented with the complaint of some fleshy red mass coming out per urethra during voiding for last 2 years. He had reddish urine 2 years back and it persisted for few days only. Suddenly he noticed some red mass protruded per urethra during voiding and it vanishes when voiding is completed. This was accompanied by decrease in urinary flow as well. The size of protruding mass also increased gradually from size of a very small grain to present size $1.5 \mathrm{X} 1 \mathrm{~cm}^{2}$ (Figure 1). There is no other significant past medical or surgical history.

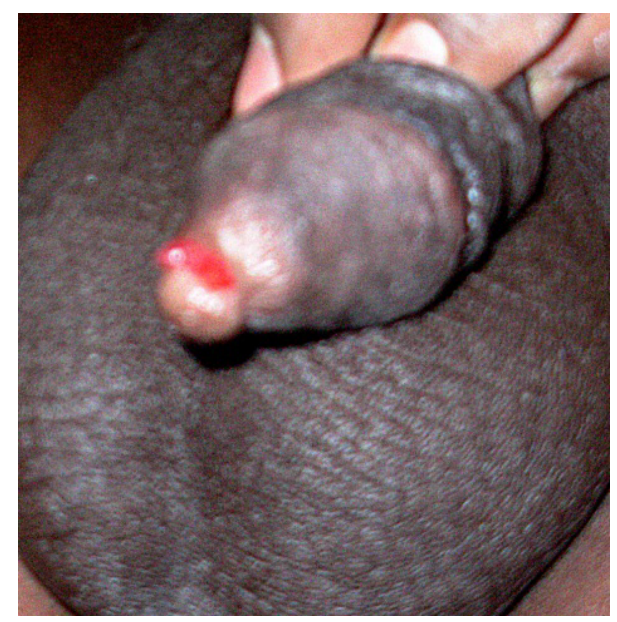

Figure 1: Prolapsing red fleshy mass from the urethra.
On examination, he had normal external genitalia with a reddish polypoidal mass measuring $1.5 \mathrm{~cm}$ from tip of the penis, coming out through external urethral meatus during valsalva maneuver, straining, voiding. Color Doppler Ultrasonography of the penis showed a highly vascular sessile mass arising from ventral part of urethra, 1.5 $\mathrm{cm}$ proximal to the meatus. His hematological and renal biochemical parameters were normal.

Under spinal anesthesia a dorsal meatotomy was done. A pedunculated red fleshy mass arising from the ventral aspect of the urethra, $1.5 \mathrm{~cm}$ proximal to the external urethral meatus was found (Figure 2). The mass was excised with low current electro-coagulation of the base and urethra was repaired on the operated area. Then cystourethroscopy was done and it revealed there was no other lesion

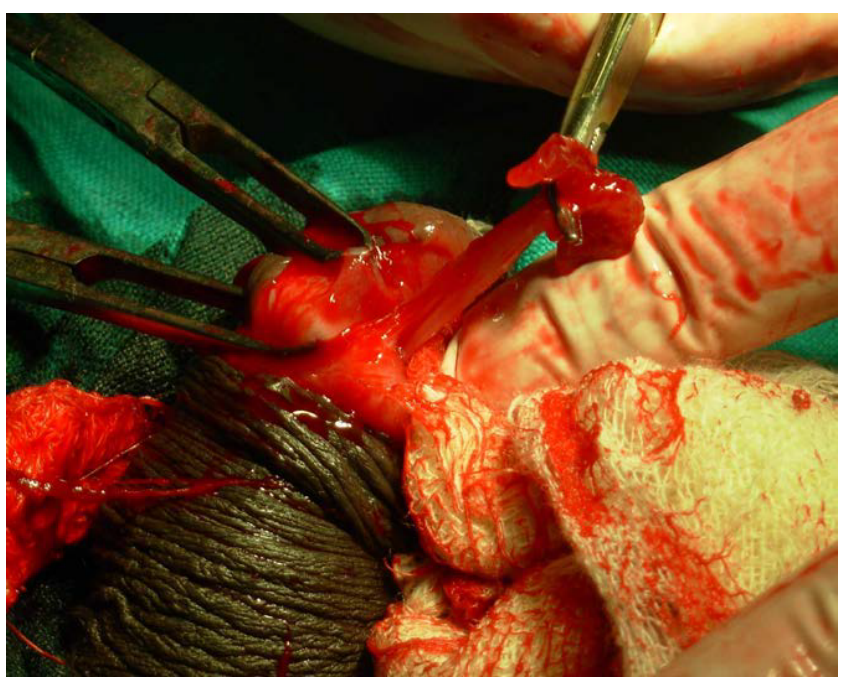

Figure 2: Per operative photograph showing a pedunculated mass arising from $1.5 \mathrm{~cm}$ proximal to the tip of urethra.

*Corresponding author: Prof. Dilip Kumar Pal, Vinayak Garden, Flat NoA/3D, 41B, Simla Road, Kolkata-700006, India, Tel: +919433132553; E-mail: drdkpal@yahoo.co.in

Received September 17, 2014; Accepted September 24, 2014; Published September 29, 2014

Citation: Pal D K (2014) Prolapsing Urethral Rhinosporidiosis. J Trop Dis 2: 151 doi: 10.4172/2329-891X.1000151

Copyright: (c) 2014 Pal D K. This is an open-access article distributed under the terms of the Creative Commons Attribution License, which permits unrestricted use, distribution, and reproduction in any medium, provided the original author and source are credited. 


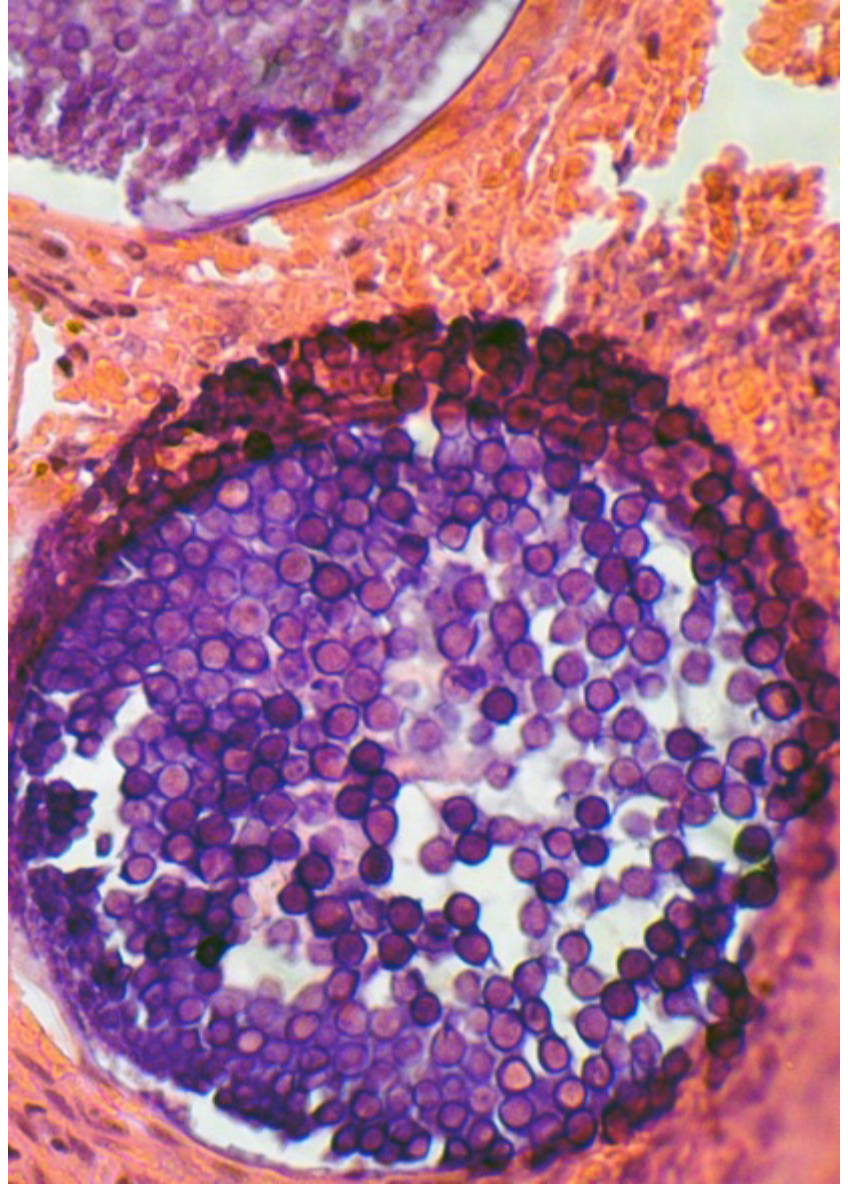

Figure 3: Histopathological microphotograph showing various stages of sporangia with neutrophilic and plasma cell infiltration.(H \& E X 400).

in the urethra or bladder. Meatotomy incision was closed leaving a catheter per urethra.

On histopathological examination of the excised mass it was found to be rhinosporidiosis (Figure 3). Cystourethroscopy was done at 3 month interval on follow up visits. Patient was doing well till 1 year without any recurrence.

\section{Discussion}

Rhinosporidiosis is a chronic granulomatous condition commonly found in India and Sri-Lanka and rarely in western world [1]. Most of the cases the patients presented from the rural area and poor socioeconomic strata $[1,5]$ as in our case. Though it is a fungal disease caused by Rhinosporidium seeberi, although its exact taxonomic classification is still in debate. It's mode of transmission is not clearly known till now. Contaminated water, dust and soil are the likely source of infection and rural background of the patients seems to substantiate this $[2,5]$. In experimental animal it is not possible to introduce the disease by inoculation and it is also not possible to culture the organism in vitro or in vivo [2]. It is not contagious there is no evidence of transmission of the disease from man to man or animal to man till now $[4,5]$.

Usually the lesion presents as a discrete, friable, painless slowgrowing polypoidal pedunculated or sessile masses which are highly vascular and bleeds on touch $[2,5]$. Nasal mucosa $(70 \%)$ and conjunctiva is the most common site of infection, but other rare sites are larynx, maxillary antrum, and skin of limbs, lachrymal sac, urethra, vagina, parotid duct, bone and rectum [4]. Urethral involvement is very rare and only few cases are reported in the literature and mostly from India $[4,5]$.

Typical clinical features of the lesion and strong suspicion lead to the diagnosis. Histopathology is the only way to confirm the diagnosis $[2,4,5]$. It reveals chronic inflammatory cell infiltrate composed of plasma cells, lymphocytes along with foreign body giant cells surrounding characteristic sporangia, seen as globular cysts of various sizes at various stages of maturity lined by well-defined chitinous wall $[1,2,4,5]$. Inside the sporangia are present numerous endospore. In our case diagnosis was made by characteristic histopathology showing sporangia and endospores amidst dense chronic inflammatory cell infiltrate.

Though the disease has benign course usually and remain localized, two fatal cases with disseminated rhinosporidiosis has been reported [4]. Surgical excission, followed by electro-coagulation of the base is the preferred method of treatment $[4,5]$ as done here at our case.Recurrence has been documented up to $25 \%$ due to inadequate resection [5]. Sometimes, partial amputation of the penis may be required, due to extensive involvement of the glans [5]. Systemic amphotericin B and Dapsone has been used with inadequate response [2].

\section{References}

1. Karunaratne WA (1964) Rhinosporidiosis in man. University of London: Athlone Press: 14-18.

2. Hangar PL (2007) Diseases of ear, nose and throat. Elsevier, 4thedn; 150-151.

3. Bhat S, Thomas A, Cherian J, Reghunath, et al. (2002) Urethral rhinosporidiosis Indian J Urol18: 188-189.

4. Sasidharan K, Subhramonian P, Moni VN, Arvindan KP, et al. (1987) Urethra Rhinosporidiosis - Analysis of 27 cases. Br J Urol 59: 66-69.

5. Pal DK, Mukherjee B, Hati GC, Chowdhry MK. (2003)Rhinosporidiosis in male urethra. Indian J Urol 19: 162-163. 